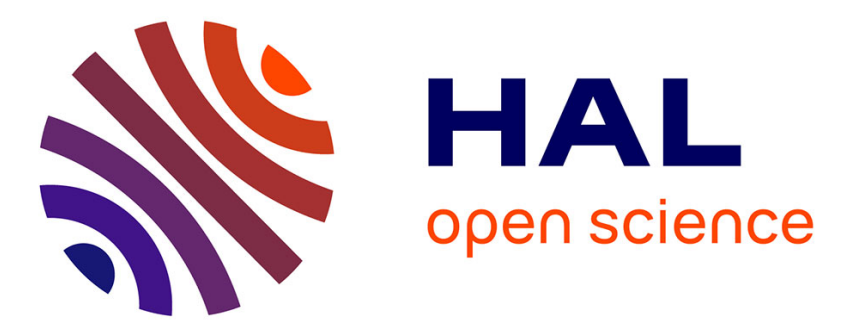

\title{
Time-frequency-phase coherence - general framework for signal analysis in passive context
}

Cornel Ioana, André Quinquis, Bertrand Gottin

\section{To cite this version:}

Cornel Ioana, André Quinquis, Bertrand Gottin. Time-frequency-phase coherence - general framework for signal analysis in passive context. IEEE PASSIVE 2008 - New Trends for Environmental Monitoring Using Passive Systems, Oct 2008, Hyeres, French Riviera, France. pp.1-11, 978-1-4244-2815-1, 10.1109/PASSIVE.2008.4787008 . hal-00448332

\section{HAL Id: hal-00448332 \\ https://hal.science/hal-00448332}

Submitted on 18 Feb 2010

HAL is a multi-disciplinary open access archive for the deposit and dissemination of scientific research documents, whether they are published or not. The documents may come from teaching and research institutions in France or abroad, or from public or private research centers.
L'archive ouverte pluridisciplinaire HAL, est destinée au dépôt et à la diffusion de documents scientifiques de niveau recherche, publiés ou non, émanant des établissements d'enseignement et de recherche français ou étrangers, des laboratoires publics ou privés. 


\title{
TIME-FREQUENCY-PHASE COHERENCE - GENERAL FRAMEWORK FOR SIGNAL ANALYSIS IN PASSIVE CONTEXT
}

\author{
CORNEL IOANA ${ }^{+}$, ANDRE QUINQUIS ${ }^{++}$, BERTRAND GOTTIN $^{+}$
}

\author{
+ : GIPSA-lab, BP 46, 961 rue de la Houille Blanche, 38402 Saint Martin d'Hères cedex, France \\ E-mail : Cornel.Ioana@gipsa-lab.inpg.fr, Bertrand.Gottin@gipsa-lab.inpg.fr \\ ${ }^{++}$: Scientific Research and Innovation Division, DET/GESMA, 29240 Brest Armées, France \\ E-mail : andre.quinquis@dga.defense.gouv.fr
}

\begin{abstract}
The characterization of a natural environment (underwater, for example) and the identification of radar/communication signals in SIGINT (signal intelligence) are just two typical examples of applications requiring signal analysis in a passive configuration. In the first case, even if the characterization is based on the analysis of received signals in an active configuration, the unknown deformations of the transmitted signal transform the signal processing problem in a passive context one. Concerning the second case, the passive behavior of the signal intelligence field is a well-known problem in the electronic warfare problem.

In this paper we propose a general signal analysis framework in passive context. We show that, in spite of the differences between some possible passive applications (underwater channel characterization and SIGINT) a unified signal analysis framework can defined. This definition starts from the general observation that real life signals received in a passive configuration are non-stationary. Their analysis in the time-frequency domain is well adapted so that it offers appropriated structures which are good candidates for the information post-processing. In a passive context, the definition of an appropriate time-frequency representation space is a complex problem, mainly related to the lack of a priori information about the processed signal. One general solution is proposed in this paper and it is based on the timefrequency-phase coherence. Conceptually, while the received signals are unknown (a model is difficult to be assumed), a general remark is the coherent shapes of their time-frequency structures. This coherence could be materialized by fundamental physical parameter of every signal - amplitude, time, frequency and initial phase.

Indeed, the signal analysis framework is defined through three blocks : detection of regions of interest, segmentation and separation, analytical characterization. This architecture is mainly based on joint use of time, frequency and local phase analysis. More precisely, the phase information will be locally analysed, using generalized instantaneous moments, on the time-frequency regions previously selected thanks to the time-frequency grouping algorithm.

This architecture constitutes an efficient scheme to solve the constraints brought by this type of signals with a complex time-frequency behavior and by the human operator to reduce his tasks in the decision process. Examples from underwater behavior (underwater mammals vocalizations) and electronic warfare will prove the efficiency of the proposed approach.
\end{abstract}

\section{INTRODUCTION}

The field of signal analysis is a very important element in a system of representation and/or information extraction. Considering the general non-stationary character of the observations encountered in real applications, their analysis in time-frequency domain constitutes the best suited technique to identify the relevant structures for information processing. There are a large number of approaches developed in timefrequency analysis field. In spite of their diversity, it is commonly used to classify the time-frequency methods as non-parametric or parametric representations. The non-parametric representations are mainly represented by the Cohen's class distribution [1]. There are numerous other works attempting to provide "nice" time-frequency visual information (see [2] for some widely followed directions). In order to better describe the time-frequency content of a given class of signals, the parametric time-frequency methods have been also introduced [2], [3]. While they are relatively efficient for a given signal class their adaptation to other signal classes is very difficult and, sometimes, even impossible. One example is the polynomial phase modeling [4] which could be difficultly applied to analyze fast-varying time-frequency structures.

A common deficiency of both types of TFRs is the marginally use of the phase information. Namely, since the large majority of the methods are mainly looking only for the instantaneous frequency law (IFL; ie, the first derivative of the instantaneous phase law), the phase law remains almost unexplored. The usual TFR ignores the phase in spite of its richness. For example, the spectrogram is a magnitude time-frequency representation. 
Recently, the phase in the time-frequency domain becomes to be studied thanks to the complementary information about the analyzed signal which is brought out [5]. The idea is straightforward : while the phase is one of the fundamental parameters of a signal, its exploitation might lead to a more efficient characterization.

In this paper we propose the joint use of magnitude time-frequency information and the phase of the signal. The work context is the extraction of time-frequency information from uncertain systems. Digital modulation identification and analysis of natural signals form both an uncertain systems since the overall properties are unknown, their estimation being the purpose of the analysis. Contrary to [4] the phase will be evaluated in the original time-domain with help of information provided by the magnitude TFR. Proceeding in this way, we show that the phase analysis will be less affected by the noise or other component than in the case of direct estimation of the phase.

The paper is structured as follows. In the section 2 we present a short overview of the conventional time-frequency tools. In the section 3 we investigate the properties of the phase in analyzing time-frequency structures. In the section 4 we describe the concept of time-frequency-phase analyzer. The results provided in section 5 illustrate the benefits of this concept. We conclude in section 6 .

\section{OVERVIEW OF CONVENTIONAL TIME-FREQUENCY ANALYSIS}

The time-frequency analysis is a very challenging research field thanks to its importance for the understanding of real-life signals. A good interpretation of a signal guarantees the efficiency of its processing. For this reason a lot of researches in signal processing concentrated their attention on timefrequency methods. It would be a very difficult task to synthesis all of works done in the past twenty years and it is not the purpose of this section. We are just proposing a general framework, starting from [6], that allows us to point out on common problems arising in this field. In the most general case any TFR of a multicomponent signal

$$
s(t)=\sum_{k=1}^{N} A_{k} e^{j \phi_{k}(t)}
$$

can be expressed as

$$
\operatorname{TFR}_{s}(t, \omega)=\sum_{k=1}^{N} 2 \pi \delta\left(\omega-\phi_{k}^{\prime}(t)\right) *_{\omega} F T\left\{\mathrm{e}^{\mathrm{j} 2 \pi \ell_{k}(t, \tau)}\right\}+\text { CrossTerms }
$$

where FT stands for the Fourier transform, $\phi_{k}^{\prime}$ is the first-order derivative of the phase law of the $k^{\text {th }}$ component of the signal (ie the IFL of this component), $* \square$ is the spectral convolution operator, $\square$ is the lag used for the computation of the TFR and $Q_{k}(t, \square)$ is a function measuring the spreading of the time-frequency energy of the $k^{\text {th }}$ component around its IFL. This function is helpful in the mono-component signal case in order to appreciate the performances of a considered TFR [6]. It measures the inner-interference terms (ie the artifact generated in the case of non-linear IFL) and, ideally, this function should be 0 .

The CrossTerms in (2) stands for the cross-terms issued from the combination of the TFRs of each possible combination of components. The majority of works in time-frequency analysis attempts to reduce the level of these terms. In any case, the consequence of such intention is the increasing of the importance of the function $Q$. Every method is subject of relative strong or weak assumptions. Very often, these assumptions transform the considered TFR in a parametric one restricting also its area of application (ie the types of signal that allow us to efficiently use the method). For this reason there is no a general method for efficient extraction of time-frequency information. This establishment is a critical issue if we cannot make any assumption. In our case, characterization of a process in a blind configureuration restricts drastically the a priori hypothesis that can be made. The existing approach in this case is to test many TFRs trying to find the "best" one, but the choice of the criterion still remains a difficult task.

The next example illustrates the problems related to the choice of a best TFR in an uncertain configureuration. In this sense, we consider two digital modulations TFRs that can be meet in a real scenario [7]. The ideal laws of modulation are given in the figure 1.a. 

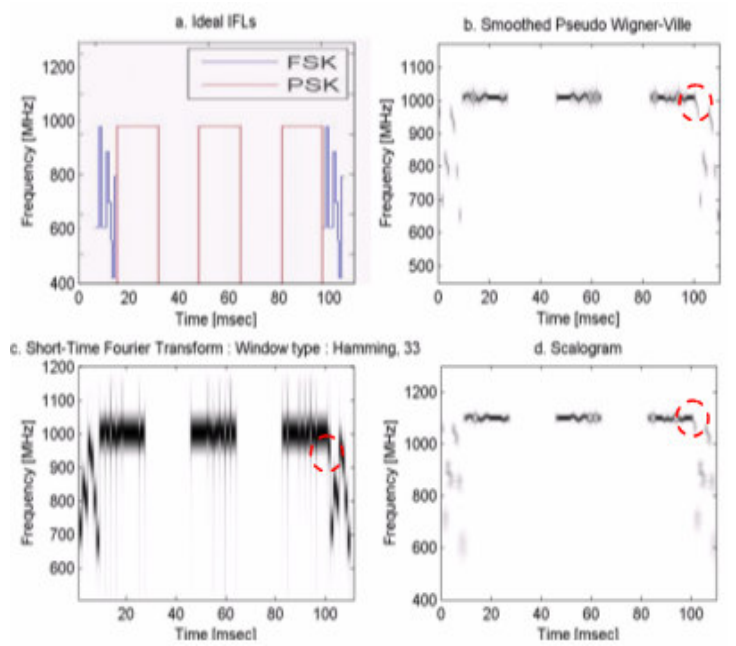

Figure 1 - Several TFRs of a mixed signal

For this mixture of two digital modulation types we show the results obtained by several well-known TFRs. In the case of Smoothed Pseudo Wigner-Ville Distribution (figure 1.b) we remark the good resolution but the phase transitions corresponding to the PSK are erased by the smoothing. These transitions are better represented in the case of the Short-Time Fourier Transform - STFT (Figure. 1.c) but the paid price is the poor resolution. For this signal, a good result seems to be provided by the scalogram (Figure. 1.d). However, all the considered TFRs provide only qualitative information about the signal. Estimation of the parameters or separation of the modulations are the kind of tasks inappropriate for these TFRs. The main reason is the representation ambiguities generated by the trade-off between resolution and artifacts level. For example, it is almost impossible to separate the third PSK packet and the first frequency step of the second FSK packet (see Figure1. b, c, d circular marker).

As shown by the expression (2) the existing TFRs use only the information provided by the first-order derivative of the phase. For this reason, the interpretation of time-frequency representation is often subject to ambiguities. To illustrate this, let us consider two different signals

$$
\begin{aligned}
& s_{1}[n]=e^{j 2 \pi\left[.1 n-2 \cdot 10^{-4} n^{2}+10^{-6} n^{3}\right]}+e^{j 2 \pi\left[.2 n-2 \cdot 10^{-4} n^{2}+10^{-6} n^{3}\right]} ; \\
& s_{2}[n]=e^{j 2 \pi\left[\varphi+.1 n-2 \cdot 10^{-4} n^{2}+10^{-6} n^{3}\right]}+e^{j 2 \pi\left[\cdot 2 n-2 \cdot 10^{-4} n^{2}+10^{-6} n^{3}\right]} \\
& \varphi=\pi / 3(\text { for } n=0, . ., 256) \text { and } \pi / 2(\text { for } n=257, . ., 512)
\end{aligned}
$$

whose effect in TFR domain is quite similar (Figure. 2).
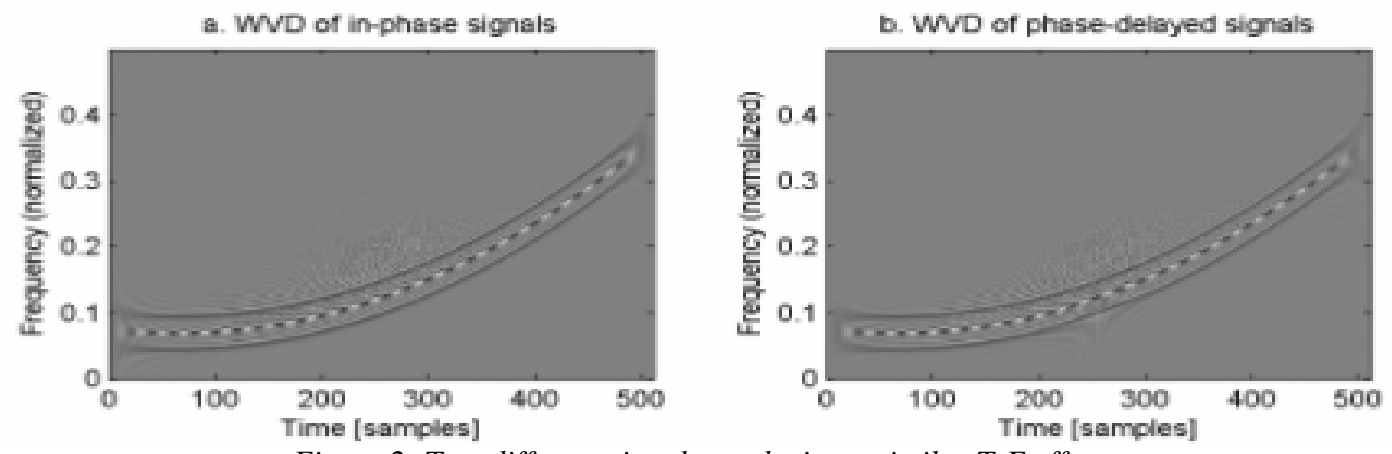

Figure 2- Two different signals producing a similar T-F effect

Obviously, the both TFRs show that the WVD is able to follow the time-frequency content variation over time. But they fail to establish the nature of each signal. One can say that the signals are of the same type but it is false as the expression (3) states.

In conclusion, the standard TFRs cannot provide the complete information about the analyzed process. A solution to solve this problem is to take into account the phase as discussed in the next section. 


\section{COMPLEMENTARITY OF TIME-FREQUENCY AND TIME-PHASE REPRESENTATIONS}

The instantaneous phase law (IPL) is a fundamental parameter of any signal, because it describes its features. It is defined as the arctangent of the ratio between imaginary and real parts of the signal (or inquadrate $\mathrm{Q}$ and in-phase I components).

$$
\hat{\phi}(t)=I P L_{s}=\arctan \left(\frac{\operatorname{Im}\{s(t)\}}{\operatorname{Re}\{s(t)\}}\right)
$$

The complete description of a signal provided by the phase is illustrated by the following example in the case of a cubic frequency modulation defined by

$$
s[n]=e^{j\left(-3.3+.31543 n-6.37 \cdot 10^{-4} n^{2}+3.14 \cdot 10^{-6} n^{3}\right)} ; n=0, \ldots, 511
$$

The WVD of this signal (Figure. 3.a) offers information about the IFL but it is affected by the "intraference" terms (inner-terms). The exact IFL is depicted in Figure. 3.c and is obtained by the derivation of the IPL. Due to the derivation, the initial phase information is lost.
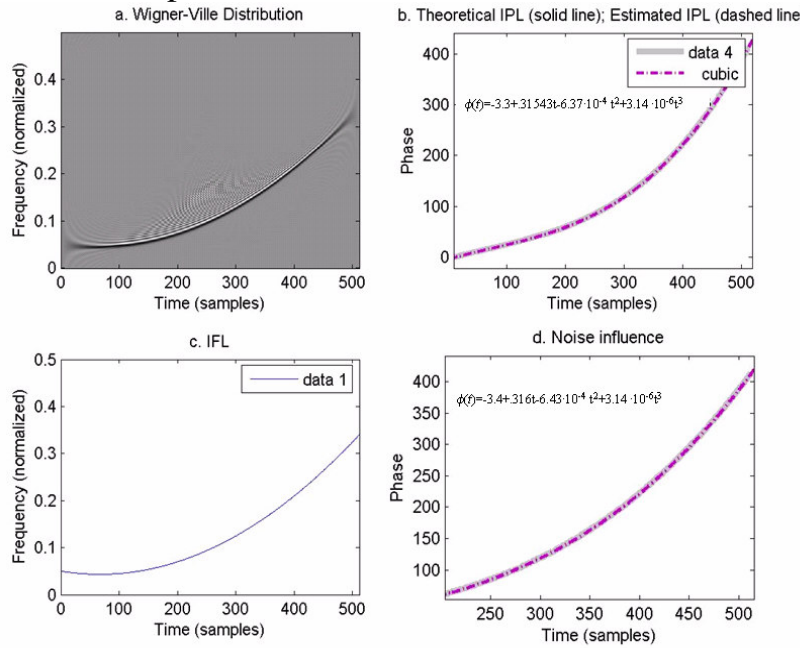

Figure 3 - Description of signal features by IPL

The IPL has been extracted by unwrapping the arctangent of Q/I ratio. As we can remark in the Figure. 3.b the polynomial phase estimation of this law provides all the signal parameters. Nevertheless, this procedure is not robust in noisy signals (Figure. 3.d) even if the noise is relatively low $(20 \mathrm{~dB})$. For this reason, the polynomial phase modeling approaches are subject of a great attention [4]. In spite of the variety of proposed methods, problems still remain especially in a multi-component signal case and when the polynomial model is not appropriate. This is the reason why we address the problem of T-F analysis in a non-parametric way (ie without any assumption about the signal type).

Example 3 shows that a signal is completely characterized by its IPL. Therefore, if the phase is appropriately used it can help us to eliminate the ambiguities in the T-F plane. Namely, the phase has to be carefully interpreted in the multi-component context. If we consider the signals (3), we remark, in Figure. 4.a, that the phases estimated via (4) contain all the information about the T-F behavior of the mixed signal but the useful information (ie the phase delay parameters) is "hidden". Fast transitions appearing in the first derivative of the phase (Figure. 4.a) are associated to the cross-terms and they mask the phase delay apparition. 


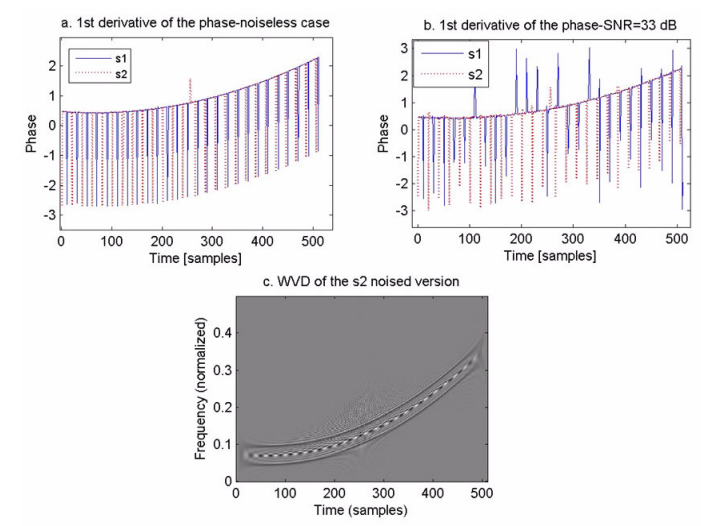

Figure 4 - Complementarity of time-frequency and time-phase information

However, since the cross-terms generate phase transitions having a regular variation, we can find the phase delay transitions by locking for irregular peaks. One might think that it is similar to the analysis of the cross-terms directly in the WVD plane (Figure. 2). Of course, the results would be similar but this procedure is very poor when the signal is embedded in noise. This is shown in Figure. 4.b : due to the noise it is almost impossible to distinguish between the cross-terms and the phase transitions (for a SNR about $33 \mathrm{~dB}$ !). Nevertheless, the TFR of this signal (Figure. 4.c) is almost untouched by the noise : the signal components are robustly represented.

Consequently, in order to find the phase information of a component, the proposed idea is to use the complementarity between the time-frequency and the time-phase representations. The concept based on this idea is addressed in the next section.

\section{TIME-FREQUENCY-PHASE ANALYZER}

As figure 3 shows the instantaneous phase includes all the information about the corresponding signal. On the other hand, figure 4 shown that analysis of the phase using the operator (4) is limited in the case of multi-component signals and even for a relative reduced noise. Complementarily, the time-frequency information is well suited for analysis of the multi-component signals and it is less sensitive to the noise. Consequently, for the analysis of the instantaneous phase of each component being part of a signal, we propose the use of the time-frequency representation and of a phase estimation operator. The choice of these items is explained as follows.

\section{A. Initial Time-Frequency Representation}

Given an unknown multi-component noised signals, the first natural operation is to represent the signal in an initial representation space (IRS). Since the unknown signal is generally non-stationary, nonparametrics TFRs are naturally the best candidates. Among the existing TFRs, everybody could choice its representation. However, since the aim is to represent the signal component, artifacts should be avoided. For this reason, the WVD is not really well advised for IRS generation purpose. Alternatively, the spectrogram is a typical example belonging to the TFR class based on a time windowing procedure. However, since this kind of procedure introduces artificial phase transitions, we prefer to avoid such process. Therefore, we build the IRS only around a filterbank-based partition of the signal's spectrum. In order to highlight the signal details we propose a Gabor-type filterbank whose transfer functions are overlapped in frequency [8]. The effect of this filterbank-processing is mathematically expressed as :

$$
\begin{aligned}
& \mathbf{W} s=\left\{s * h_{k} \mid k=1, . ., N_{\text {fithers }}\right\} \\
& h_{k}(t)=\operatorname{IFFT}\left\{e^{-2 \pi^{2} \sigma^{2}\left(f-f_{k}\right)^{2}}\right\}
\end{aligned}
$$

where IFFT stands for the Inverse Fourier transform.

The interest of the filterbank analysis-based concept is obvious. Since the analyzed signal has generally a complex T-F structure, its representation in several sub-bands leads to a reduction of the representation complexity. Analyzing a signal in a given sub-band and around its neighborhood allows us to identify signal structures much easier than searching in all T-F plan. As identification criterion, we can use the local energy criterion as proposed in [9], for example. The idea is to depict time-frequency structures whose energy is higher than a local threshold and that are composed by contiguous energy atoms. Hence, given two atoms $s_{1}$ and $s_{2}, s_{1}, s_{2} \subset \mathbf{W} s$ having partially overlapped time-frequency supports, we generally 
associate them to the same component $\boldsymbol{c}$ if one, part of or all the following conditions are satisfied :

$$
\mathbf{E} s_{1}>\varepsilon \wedge \mathbf{E} s_{2}>\varepsilon
$$

where $\mathbf{E}$ stands for the energy and $\varepsilon$ is a threshold computed locally ;

$$
\sqrt{\left(t_{s 1}-t_{s 2}\right)^{2}+\left(f_{s 1}-f_{s 2}\right)^{2}} \leq \gamma
$$

where $\left(t_{\mathrm{si}}, f_{\mathrm{si}}\right)$ are the time and frequency centers of the signals $s_{i}(i=1,2)$ and $\gamma$ is a time-frequency distance threshold;

$$
\left|\frac{d \mathbf{E} s_{1} \Theta s_{2}}{d \theta}\right| \leq \eta
$$

where $\mathbf{E} s_{1} \Theta s_{2}$ is the time-frequency energy comprised between $s_{1}$ and $s_{2}, \eta$ is threshold measuring the degree of energy continuity. If the gradient of the energy between the both atoms is below a certain threshold, one can conclude that both atoms belong to the same component $\boldsymbol{c}$.

The use of these criteria allows associating the time-frequency atoms as suggested in the next figure.

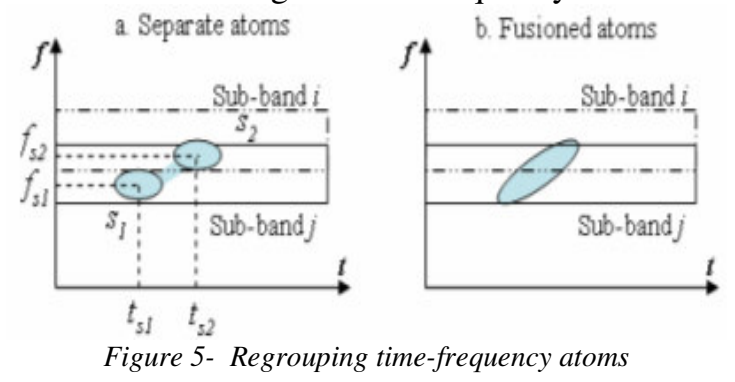

The both atoms illustrated in Figure. 5.a could be merged with help of criteria (7)-(9) - Figure. 5.b. The choice of criteria (7)-(9) is a matter of time-frequency regrouping strategy which is selected according to the application (see [1], [2], [8], [9] for some existing methods).

There are a large number of works combining criteria (7)-(9) and filterbank analysis. However, there are situations when the criteria (7)-(8) are limited. Such a case arises when the atoms are too far in timefrequency plane. Energy is between the atoms is spread out many sub-bands and they cannot be regrouped. This is illustrated for the signal used in the figure 1 (Figure. 6.a).

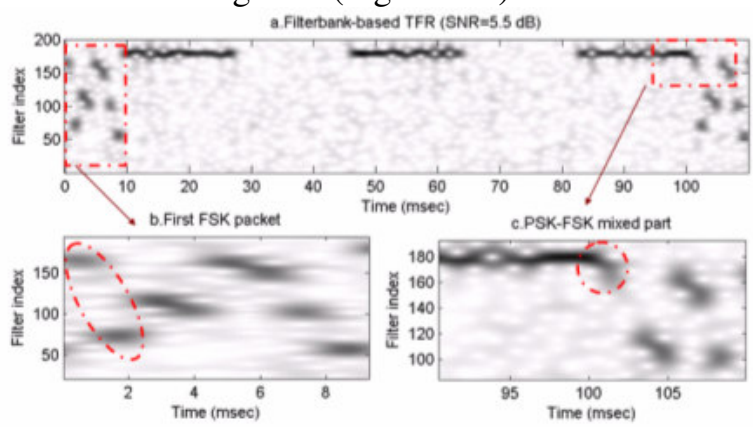

Figure 6 - Limitations of classical T-F regrouping criteria

As we observe in Figure. 6.b the two encircled atoms of the FSK are too far to be associated by the criteria (8) and (9). In the same time (Figure. 6.c) the last part of the PSK and the first atom of the FSK will be associated since they are very close. This kind of situation will be solved by the phase evaluation in an appropriate way.

\section{B. Phase analysis operator}

We saw previously that the time-frequency IRS provides robust T-F information about the analysis process but it remains deficient when the components are too far (the case of the fast modulations) or too close (in this case, the finite resolution of the filterbank acts). A possible solution to eliminate the ambiguities is to apply the phase operator analysis (defined, for example, by (4) but other methods could be imagined [10]). As we explained in the section 3, the direct application of the operator (4) is not judicious because of either interferences either the noise. The idea we propose is to apply this operator only for the 
signal corresponding to the time-frequency region we are about to analyze it. In the same time, in order to avoid the noise and the artifacts as much as possible, it is necessary to define this region in an appropriate manner. Intuitively (see Figure. 7), the design of the region around the connection line of the atoms is more appropriate than the simple rectangular definition. In other words, with help of the IRS, we can define the T$\mathrm{F}$ region according to the content of data inside it.

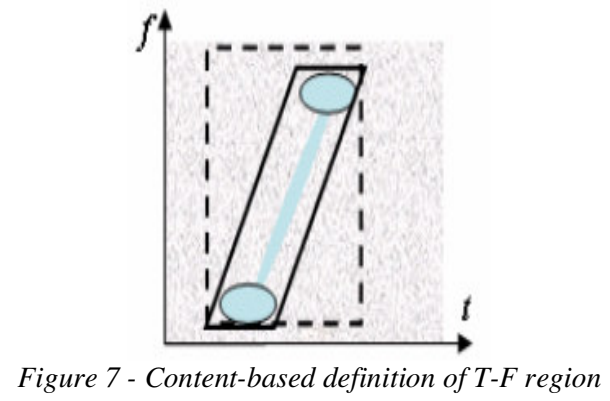

This content-based design of T-F region $\mathbf{R}$ allows us to define the parameters of a time-frequency filter. The method we used is based on the non-unitary time-warping concept [11]. Namely, the filter is designed by analogy of the FIR methodology but for a time axis that follows the nature of the region. The result will be an impulse response $h_{w}$ that matches very efficiently the features of the signal bounded by $\mathbf{R}$. Applying this filter on the original signal $s$ is equivalent with the extraction of the signal located inside this region :

$$
s_{\mathbf{R}}=s * h_{w}
$$

Since the ultimate goal of this operation is the estimation of the phase via (4), we impose that the filter $h_{w}$ be able to conserve the phase feature of the original signal. An interesting idea is device in [11] where a forward-backward strategy is used in order to design filters with zero phase characteristic. That is, thanks to [11], we can extract correctly the signal corresponding to two or more detected atoms in the IRS. Let denote with $\phi_{\mathbf{R}}$ the phase of the signal $x_{\mathbf{R}}$. For this function we can define the continuity concept in the considered time-frequency region. Mathematically, this could be done with help of singularity concept. In our context, the continuity concept could be used in the following way. If the phase $\phi_{\mathrm{R}}$ contains one or many singularities it means that the phase of the signal $x_{\mathbf{R}}$ has fast variation. This is typical for atoms belonging to distinct components. Alternatively, if the phase is slowly varying we can conclude that the atoms belong to the same component. Mathematically, the concept of phase-based continuity test can be formulated as follows.

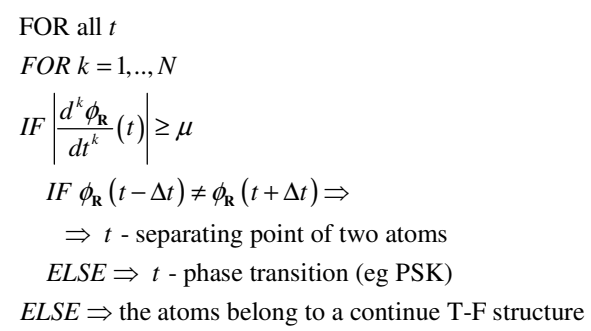

where $\square$ is a threshold applied in the $k^{\text {th }}$-order derivative time-phase domain. Note that if the atoms are simple Gabor atoms ones (eg. PSK or FSK components) the first order derivative is sufficient to apply this continuity criterion.

Both time-frequency and time-phase analysis concepts form the time-frequency-phase (T-F-Ph) analyzer whose general structure is depicted in the next figure.

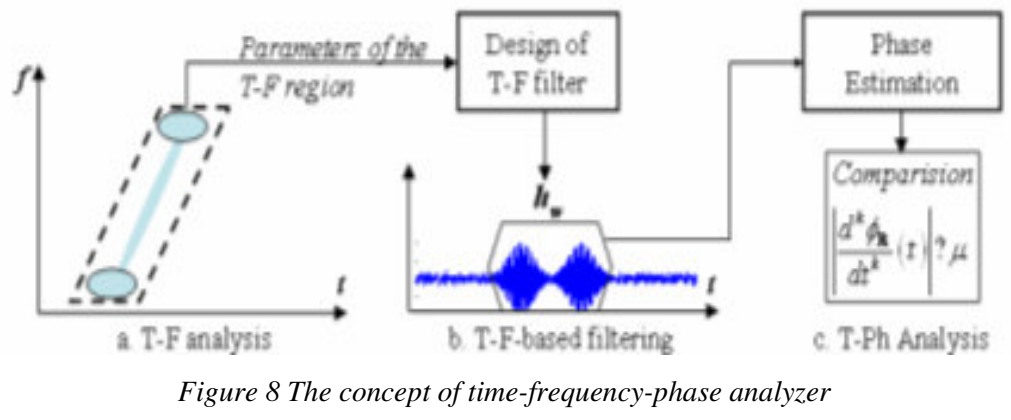




\section{RESULTS}

In this section, we illustrate how the time-frequency-phase analyzer performs and avoid the ambiguities existing in the case of a simple time-frequency analysis. For these purposes we consider the previous examples. Let consider the two-component signal defined by (3). In order to take advantage on the complementarity of the time-frequency and time-phase representations, we apply, according to the figure. 8 , a time-frequency filtering procedure as illustrated in the figure 9.b.
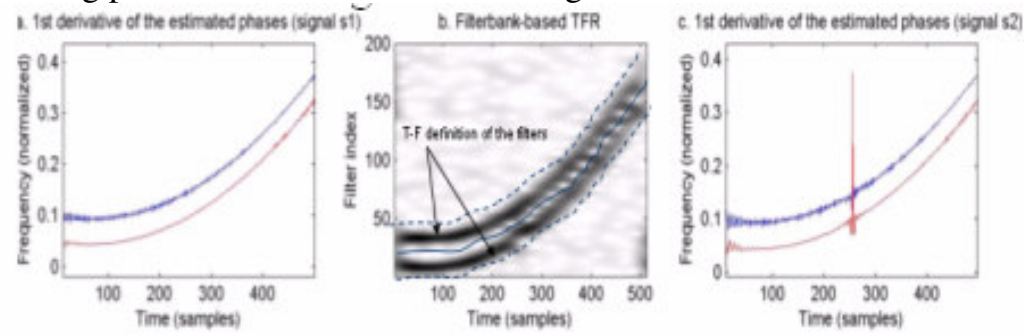

Figure 9 T-F-Ph analysis of signals defined by (3)

The signals issued by filtering as shown in Figure. 9.b. are analyzed with the phase analysis operator (4). Since the both components are separated the phase is correctly estimated. Hence, we can clearly distinguish the differences between the both signals. In the same time we obtain all the details about the phase of each component (Figure. 9.a, c) : the time-frequency shapes are correctly estimated as well as the phase transition (Figure. 9.c).

Another example consists in segmentation of the FSK-PSK signal defined in Figure. 1. As it is shown in the Figure. 10, using the T-F-Ph analyzer we can correctly separate the components of each modulation.

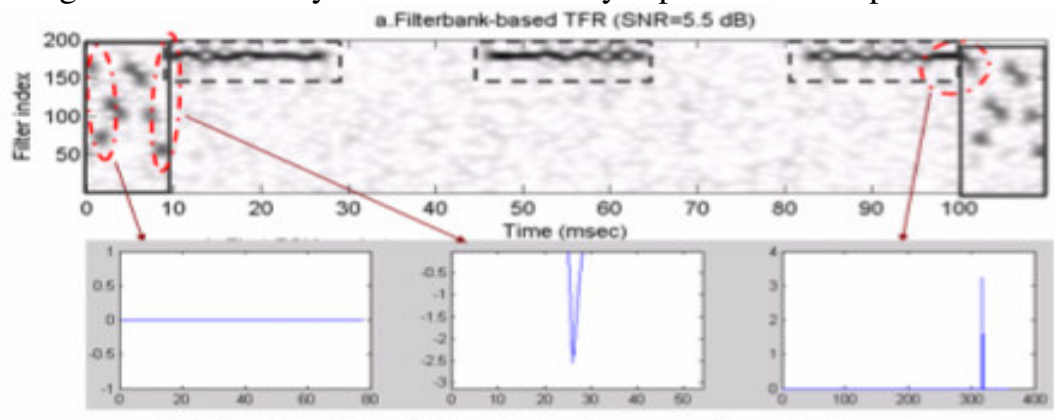

b. First derivatives of the estimated phases in the encircled regions Figure 10 Segmentation of a FSK-PSK composite signal

In order to prove the richness of the phase information we illustrate the phase of three "tricky" regions. The first consists of two FSK atoms; as we can remark, the $1^{\text {st }}$ phase derivative is constant which means that both atoms belong to the same signal. In the second case, we consider the last atom of the FSK and the first PSK packet. In this case, the phase discontinuity indicates that both parts of signals do not belong to the same one. The same establishment for the third case : in spite of the proximity between components, the phase discontinuity shows unambiguously that there are two different signals. In the same time the classical TFRs (see Figure. 1) are not able to associate these atoms to the right component.

Applying the T-F-Ph analyzer we successfully segment the signal according to the modulation types. Furthermore, this benefit will allow us to estimate the features of each modulation very important task in the field of signal intelligence (SIGINT) [7]. Otherwise, the case illustrated in Figure. 10 can arise in active communication or radar field. Due to the parasite communication signals or artifacts, the pre-processing device of receiver could be subject of wrong detection. This error can be felt further, during the adaptive filtering process. In conclusion, in the domain of communications in non-cooperative and/or signal-sense high density environment, the T-F-Ph analyzer constitutes a potential solution.

Another application concerns the time-frequency modeling of underwater mammal vocalizes. The next figure illustrates the benefits of the time-frequency-phase concept in the case of a real signal emitted by a marine mammal recorded in Bay of Biscay [12]. 

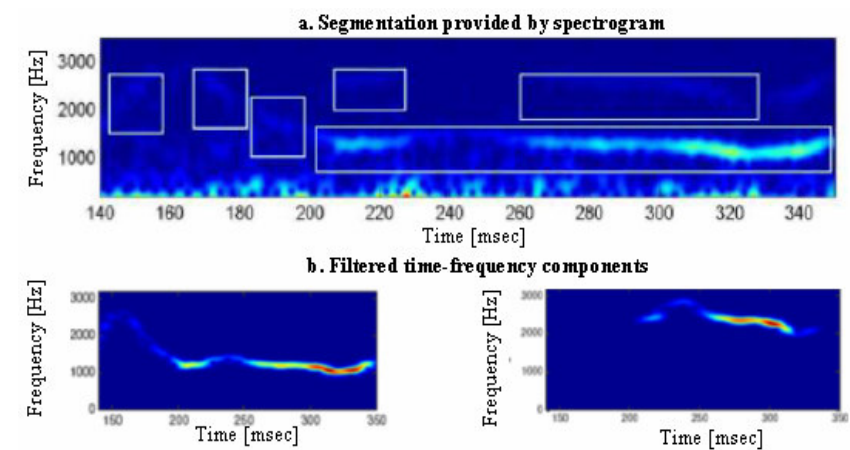

Figure 11 Time-frequency filtering based on time-frequency-phase information

The segmentation of time-frequency components issued from the spectrogram (figure 11.a.) is not correct since the time-frequency energy is varying. This is generally the case of real signals. In order to solve this problem the time-frequency-phase concept is successfully used as indicated by the figure 11.b : thanks to a correct representation of the time-frequency components, their filtering is successfully done.

\section{CONCLUSION}

The purpose of this paper was to show how the time-frequency and the time-phase informations could be used jointly in order to solve difficult situations, untrateable by the convetional time-frequency concepts. Conceptually, phase information will work towards avoiding the ambiguities by defining the notion of continuity of time-frequency structure. It can help us to solve situations when the conventional T-F analysis falls. For example, it allows us to connect components belonging to the same structure even if the TFR is affected by any type of artifacts (cross-terms, noise, attenuation, etc.). The joint use of conventional timefrequency representations and the phase of the signal constitutes the background of the time-frequencyphase analyzer.

Of course, the methods we used in this paper to implement the T-F-Ph analyzer are just sugestions. Everybody could use his own methods. Concerning our future works, two axes will be addressed. The first one deals with the generalized definition of the initial representation space. The second one consists in analyzing and proposing more efficient phase analysis operators, especially from noise robustness and multicomponent point of view.

\section{REFERENCES}

[1] L. Cohen , Time-Frequency Analysis, Pretince Hall, New Jersey, 1993.

[2] A.Papandreou-Suppappola, ed., Applications in time-frequency signal processing, CRC Press, Boca Raton, 2003.

[3] Y. Grenier, Parametric Time-Frequency Representations, in Signal Processing, eds. J.L. Lacoume, Les Houches, Session XLV, Amsterdam, vol. 1, pp. 339-397, 1987.

[4] C. Ioana, A. Quinquis, Time-Frequency Analysis using Warped-Based High-Order Phase Modeling, EURASIP Journal of Applied Signal Processing, 2005(17):2856-2873, Sept. 2005.

[5] F. Léonard, Phase spetrogram and frequency spectrogram, Traitement du Signal, vol. 17, n 4 , pp. 269-286, 2000.

[6] S. Stankovic, L. Stankovic, Introducing time-frequency distributions with a complex time arguments, Electronic Letters, vol. 32, No. 14, pp. 1265-1267, July 1996.

[7] P.E. Pace, Detection and Classifying low Probability of Intercept Radar, Artech House, Norwood, 2004.

[8] A. Teolis, Computationnal Signal Processing with Wavelets, Birkhäuser, Boston, 1998.

[9] ] C. Cornu et all, Time-frequency detection using Gabor filter banks and Viterbi based grouping algorithm, Proceedings of IEEE International Conference on Acoustic, Speech and Signal Processing ICASSP 2005,Vol 4, pp. 499-500, Philadelphia, USA.

[10] C. Ioana et all, Analysis Of Time-Frequency Transient Components Using Phase Chirping Operator, IEEE International Conference on Acoustic, Speech and Signal Processing ICASSP 2006, Toulouse.

[11] A. Jarrot, C. Ioana, A. Quinquis, A class of time-frequency filters based on non-unitary time-warping operators, Paper submitted to IEEE Transaction on Signal Processing, March 2006.

[12] A. Quinquis, C. Ioana, A. Jarrot, C. Cornu, Identification et reconnaissance des signaux et systèmes sous-marins non stationnaires, Conférence Caractérisation du Milieu Marin, Brest, Octobre 2006. 